\title{
Correction to: Impaired endogenous nighttime melatonin secretion relates to intrarenal renin-angiotensin system activation and renal damage in patients with chronic kidney disease
}

\author{
Sayaka Ishigaki ${ }^{1}$. Naro Ohashi ${ }^{1}$ - Shinsuke Isobe ${ }^{1} \cdot$ Naoko Tsuji $^{1} \cdot$ Takamasa Iwakura $^{1}$ - Masafumi Ono ${ }^{1}$. \\ Yukitoshi Sakao ${ }^{2} \cdot$ Takayuki Tsuji $^{1}$ - Akihiko Kato ${ }^{2} \cdot$ Hiroaki Miyajima ${ }^{1} \cdot$ Hideo Yasuda ${ }^{1}$
}

Published online: 13 December 2018

(c) Japanese Society of Nephrology 2018

\section{Correction to: Clin Exp Nephrol (2016) 20:878-884 https://doi.org/10.1007/s10157-015-1224-x}

In the original publication, an error occurred in Table 4(B), under Nighttime group. The value of "Nighttime Log U-AGT/Cr" for model 3 (under $R=0.68$ ) was incorrectly published as 0.11 . The correct value should read as -0.31 .

The corrected table is given below.
The original article can be found online at https://doi.org/10.1007/ s10157-015-1224-x.

\section{Naro Ohashi}

ohashi-n@hama-med.ac.jp

1 Internal Medicine 1, Hamamatsu University School of Medicine, 1-20-1 Handayama, Higashi-ku,

Hamamatsu 431-3192, Japan

2 Blood Purification Unit, Hamamatsu University School of Medicine, 1-20-1 Handayama, Higashi-ku, Hamamatsu 431-3192, Japan
Table 4 Multiple regression analyses for urinary 6-sulphatoxymelatonin per hour (U-aMT6s/hr) levels by age, gender, estimated glomerular filtration rate (eGFR), and one of the parameters \{blood pressures (BPs), urinary angiotensinogen/ creatinine ratio (U-AGT/Cr), or urinary albumin/creatinine ratio (U-Alb/Cr) \} during daytime and nighttime 
B. Nighttime

\begin{tabular}{|c|c|c|c|c|c|c|c|c|}
\hline & \multicolumn{2}{|l|}{ Model 1} & \multicolumn{2}{|l|}{ Model 2} & \multicolumn{2}{|l|}{ Model 3} & \multicolumn{2}{|l|}{ Model 4} \\
\hline & $R=0.66$ & $P<0.001$ & $R=0.65$ & $P<0.001$ & $R=0.68$ & $P<0.001$ & $R=0.69$ & $P<0.001$ \\
\hline & $\beta$ & $P$ value & $\beta$ & $P$ value & $\beta$ & $P$ value & $\beta$ & $P$ value \\
\hline Age & -0.069 & 0.63 & -0.11 & 0.42 & -0.075 & 0.58 & -0.083 & 0.53 \\
\hline Gender & -0.009 & 0.94 & -0.041 & 0.72 & -0.11 & 0.32 & -0.025 & 0.82 \\
\hline eGFR & 0.50 & 0.002 & 0.55 & 0.001 & 0.38 & 0.024 & 0.52 & $<0.001$ \\
\hline $\begin{array}{l}\text { Nighttime sys- } \\
\text { tolic BP }\end{array}$ & -0.16 & 0.27 & & & & & & \\
\hline $\begin{array}{l}\text { Nighttime dias- } \\
\text { tolic BP }\end{array}$ & & & -0.046 & 0.72 & & & & \\
\hline $\begin{array}{l}\text { Nighttime log } \\
\text { U-AGT/Cr }\end{array}$ & & & & & -0.31 & 0.044 & & \\
\hline $\begin{array}{l}\text { Nighttime log } \\
\text { U-Alb/Cr }\end{array}$ & & & & & & & -0.25 & 0.025 \\
\hline
\end{tabular}

